\title{
Chimiscope: A Multi-faceted Laboratory Platform for a Unique Journey into (Bio) molecular Science
}

\author{
Didier Perret*
}

\begin{abstract}
The University of Geneva, its Faculty of Science, and its School of Chemistry and Biochemistry have created the Chimiscope, a show to intrigue and to incite interest in the science of molecules and biomolecules. The Chimiscope will be inaugurated on the 24th November 2011.
\end{abstract}

Keywords: Chemistry education · Chimiscope · University of Geneva

The International Year of Chemistry 2011 offers a unique opportunity to stimulate interest among the general public and young people. It is well known that there is a continuing shortage of MINT (mathematics, informatics, natural and technical sciences) specialists in Switzerland ${ }^{[1]}$ and in most countries with a high wealth index, and the gap between the demand for specialists from industry and the number of graduate students is also found in the chemical and pharmaceutical industries. The Geneva area, where there are internationally renowned industries, is no exception.

With this in mind, the University of Geneva, its Faculty of Science, and its School of Chemistry and Biochemistry have decided to create the Chimiscope, a show to intrigue and to incite an interest in the science of molecules and biomolecules. The Chimiscope has been designed in a similar way to the Physiscope, ${ }^{[2]}$ an initiative of the University of Geneva and the National Center of Competence in Research MaNEP (Materials with Novel Electronic Properties), which opened in 2008 for students aged 13-18 and which has acquired an increasing audience since

\footnotetext{
${ }^{\star}$ Correspondence: Dr. D. Perret University of Geneva

School of Chemistry and Biochemistry

30, quai Ernest-Ansermet

$\mathrm{CH}-1211$ Geneva 4

Tel.: +41223796024

Fax: +41223796023

E-mail: chimiscope@unige.ch

Web: http://chimiscope.ch
}

then (4200 visitors in 2010-2011; 8700 visitors since 2008). The Chimiscope is financially and scientifically supported by the University of Geneva, the Association Industrielle Genevoise des Sciences de la Vie (AIGSV), and several chemical and pharmaceutical industries in Geneva and in Switzerland.

The Chimiscope will be inaugurated on the 24th November 2011 in presence of the teaching staff and of the political authorities of Geneva.

The main aim of the Chimiscope is to throw an attractive and stimulating light on the basic concepts that drive the world of molecules, then to show their applications to our macroscopic world, and to demonstrate the crucial role of modern chemistry and biochemistry in our society. The target audience of the Chimiscope is primarily pupils and students aged 10-18, but places will also be available for the general public. To achieve this aim and to encompass the broadest range of topics related to molecules and biomolecules, a series of six modules have been designed:

Green enlightening chemistry ('Chemistry and energy'): This topic discusses the contributions of chemistry to the search for future alternate sources of energy, with a special emphasis on the new generations of photovoltaic cells and the efficient production/storage/use of hydrogen, starting from the fundamentals (charge separation by light excitation and electron-proton coupled charge transfer in molecular systems, novel light-harvesting molecular devices) to reach the global problem of the worldwide energy demand.

What would be life without a touch of colour? ('Chemistry and colours') This topic introduces electronic energy levels, waves, light and colour; it makes use of lasers and minispectrometers for manipulating the concepts of light absorption, complementary colours, and light emission at the molecular level. Analytical spectrometry is also presented in this module as a qualitative and quantitative tool for the identification of unknown species in complex mixtures.

Let's twist with molecular motors ('Chemistry of life'): This topic addresses the properties of bioorganic materials that constitute living species; in particular, it is demonstrated that the protein myosin can transform the energy of chemical bonds (e.g. hydrolysis of ATP) into mechanical work, thus behaving as a nanomachine or a molecular motor within cells. Speeds, forces and efficiency rates of such processes can be accessed from simple microscopic observations.

Chemical brothers ('Chirality'): This topic demonstrates the basics of chirality and the principles of structure-activity relationships of organic compounds, and their crucial consequences in chemical, biological and pharmaceutical applications; the effect of chiral centres on polarised light is stressed, as well as the use of modern chromatography for the separation of mixtures of racemates. Geneva being home of the two world leaders in perfumes (Firmenich and Givaudan), chiral fragrance-active molecules are obvious actors in this module.

Living in a cleaner environment ('Chemistry and aquatic environment'): Water is ubiquitous on Earth but it is a fragile resource under human pressure; this topic highlights the biogeophysical cycles that drive aquatic ecosystems and the effect of foreign organic and inorganic substances on the quality of natural waters. Chemical and biological water treatment methods are presented and modern analytical tools are used to identify trace levels of pollutants.

Home, sweet chemical home ('Chemistry at home'): This topic illustrates, by means of selected examples, what 


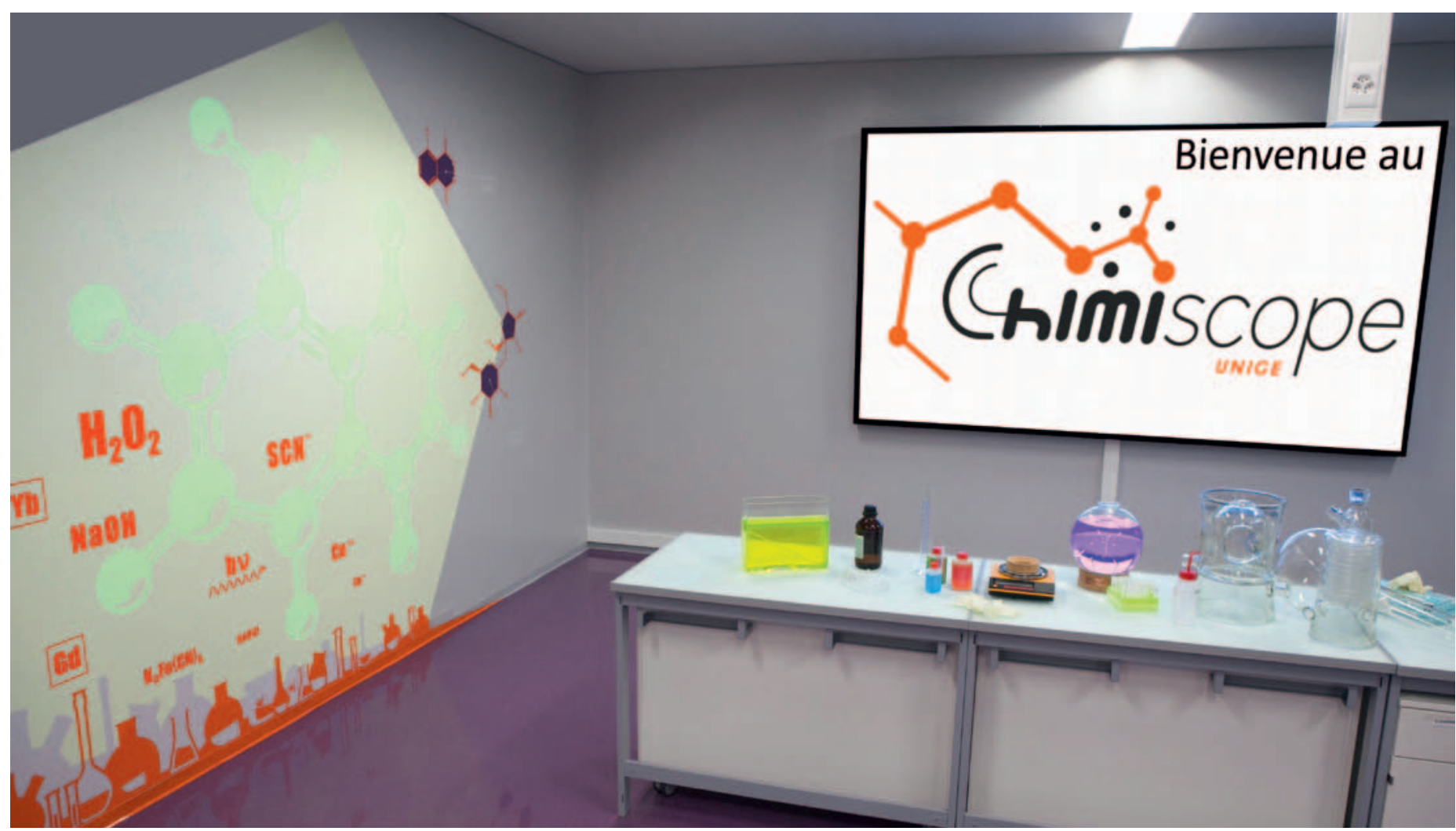

Partial view of the $100 \mathrm{~m}^{2}$ lab space of the Chimiscope, showing the interactive wall of screens allowing small-scale experiments to be displayed to a whole class; when light is dimmed in the lab (e.g. for experiments on luminescence), the decorative molecule to the left, showing the structure of luminol, becomes visible due to its phosphorescent paint. The lab is equipped with the latest equipment, including a mobile fumehood with $360^{\circ}$ vision. Photo: Jacques Erard, University of Geneva.

chemistry - of natural or artificial origin brings to our everyday life. In this module, a virtual tour of an apartment is the pretext for introducing the basic concepts of acid-base, hydrophilicity-hydrophobicity, polymerisation and plastics, tensioactive compounds, thermodynamics of cooking, inks, dyes and paints, and pharmacoactive molecules.

Modules 1 and 2 will be offered to visitors from the inauguration of the Chimiscope, and modules 3-6 will be introduced gradually. It is planned to create additional modules in the near future, particularly in the field of pharmaceutical science. All these modules are conceived with the support of high-school science teachers, in order to harmonise with the official chemistry programmes given in class.

In practice, visitors will book their 1-hour visit via the Chimiscope website. After a short welcome and an introductory presentation in a special lecture room, visitors will move to the heart of the Chimiscope, the lab space. There, handson experiments will be performed by visitors while demonstrations requiring more skill will be presented by animators trained in teaching and science outreach. The presentation accompanying the experiments will be adapted according to the age and background level of the audience.
It is hoped that an increasing number of students will be motivated to undertake a scientific career by their visit to the Chimiscope.

Received: October 8, 2011

[1] 'Pénurie de spécialistes MINT en Suisse', Rapport du Conseil Fédéral, 2010 and references therein.

[2] C. Renner, 'Hands-on inspiration for science', Nature Materials 2009, 8, 245. 